\title{
Incertitudes et confiance, une question de pratique
}

\section{Marianne Rychner}

Dre rer. soc., enseignante dans différentes HES

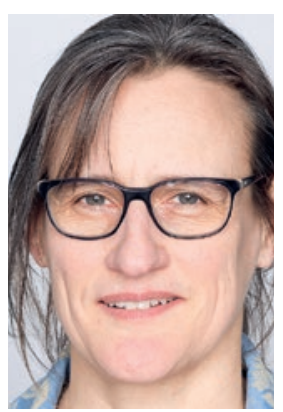

Le rapport entre la médecine en tant que science et la pratique médicale paraît simple: on étudie d'abord la matière à l'université, puis on l'applique. La réalité étant toutefois plus compliquée, il faut souvent clarifier ce lien afin d'éviter les malentendus récurrents en politique de santé, notamment dans le cadre des débats controversés sur le coronavirus. Il s'agit par exemple de savoir, en Allemagne surtout, quel virologue a raison, tandis qu'en Suisse, on se pose la question de l'utilité du masque et se demande si on peut prendre ses petits-enfants dans ses bras.

Répondre de manière incertaine à ces questions est souvent très mal accepté, car des certitudes et des consignes claires sont attendues. Or ceci ne reflète pas l'image de la science. Il est irréaliste de la voir comme un ensemble de faits à la fois hautement actuels et dépourvus de toute contradiction, alors que c'est justement grâce aux incertitudes qu'on améliore les connaissances. Il se peut que les désillusions face aux solutions espérées aient servi de terreau fertile aux théories du complot et aux détracteurs de la science.

Alors qu'il est absolument nécessaire de peser le pour et le contre et d'avancer des probabilités et non des certitudes, cette mise en balance est perçue et dépeinte comme une lacune. C'est précisément là que la science abstraite laisse la place à la pratique médicale - ce qui n'est pas une lacune, mais une chance.

La théorie sociologique de la professionnalisation s'intéresse à la question de la mise en pratique de la science dans les métiers où il existe un contact avec la clientèle: à la différence des métiers techniques, les médecins ne peuvent et ne doivent jamais se contenter d'appliquer leur expertise de manière standardisée. Ils prennent en charge des personnes avec une histoire, une volonté propre, une approche spécifique de la santé et de la maladie et un environnement de vie. Avec l'expérience, un médecin tient compte de cette complexité pour établir le diagnostic et le traitement. Le patient peut donc faire confiance au médecin: ce que celui-ci suggère est - vraisemblablement - ce qui est le plus approprié. Cette démarche holistique, qui ne se contente pas de catégoriser à la hâte mais évalue les besoins précis d'une personne, exige un certain degré d'autonomie dans l'exercice de la profession, et non des directives contraignantes. Le bon fonctionnement du système de santé en dépend - ce qui est souvent négligé lorsque des décisions sont prises en politique de santé. Par exemple lorsque la pratique médicale est dictée par des mécanismes de marché ou la bureaucratie. Ces deux formes de "contrôle» ne rendent pas justice à la logique interne de l'activité, parce qu'elles suggèrent, chacune à leur manière, que cette activité est mesurable alors qu'elle ne peut l'être, que ce soit à travers des directives répressives, la technocratie ou l'adaptation de processus en faveur d'un produit conforme au marché.

L'autonomie médicale se nourrit donc toujours de faits aux significations multiples, qui ne prennent leur sens que dans l'interprétation du cas individuel. Ce n'est pas un hasard si le terme allemand «Praxis» se réfère aussi bien au cabinet médical qu'à la pratique par opposition à la théorie - qu'il s'agisse de petits bobos ou de drames existentiels. Cela signifie aussi que pour un même diagnostic, le traitement sera différent d'une personne à l'autre: physiothérapie dans un cas, cortisone dans le deuxième et opération dans le troisième, voire des granulés homéopathiques à l'efficacité controversée. Le placebo est à chaque fois inclus, à plus ou moins forte dose. En sociologie, l'approche de la théorie de la professionnalisation décrite ici est souvent accusée de fournir aux médecins l'idéologie qui leur permet d'agir dans leur propre intérêt. C'est peut-être vrai parfois. J'estime pourtant que la tendance inverse est au moins aussi problématique, soit lorsqu'une approche standardisée est non seulement souhaitée au niveau politique, mais aussi par les médecins eux-mêmes, notamment quand il est question de rationner les prestations médicales. Un exemple récent: le débat sur la limite d'âge pour le traitement en soins intensifs des patients atteints du Covid-19. Le patient ne peut pas se fier à des chiffres ni à des listes arbitraires; il accorde sa confiance à l'humain. Et en matière de santé et de maladie, la confiance est cruciale, qu'il s'agisse de guérison ou de fin de vie dans la dignité. 